\title{
Adverse drug reactions causing treatment change among patients taking highly active antiretroviral therapy in health care facilities of Mekelle, Ethiopia
}

\author{
Abebe Ejigu', Mussie Gehzu², Werissaw Haileselassie ${ }^{3}$ \\ 'Department of Pharmacology and Clinical Pharmacy, School of Pharmacy, Addis Ababa University, Addis Ababa, Ethiopia. \\ ${ }^{2}$ Department of Pharmacy, College of Health Sciences, Mekelle University, Mekelle, Ethiopia. \\ ${ }^{3}$ Department of Reproductive Health and Health Service Management, School of public health, Addis Ababa University, Addis Ababa, Ethiopia.
}

\begin{tabular}{|c|c|}
\hline ARTICLE INFO & ABSTRACT \\
\hline $\begin{array}{l}\text { Article history: } \\
\text { Received on: } 15 / 11 / 2017 \\
\text { Accepted on: 04/02/2018 } \\
\text { Available online: } 30 / 03 / 2018\end{array}$ & $\begin{array}{l}\text { Adverse drug reactions to antiretroviral drugs have been identified as one of the major cause for non-adherence and } \\
\text { change in regimen that in turn affects treatment outcomes negatively. This study was undertaken to determine the } \\
\text { prevalence of adverse drug reactions to highly active antiretroviral therapy that result in change in regimen in two } \\
\text { health care facilities (MH and MHC) in Mekelle, Ethiopia. A cross sectional retrospective study was conducted on a } \\
\text { total of } 3304 \text { HIV patients currently on HAART, } 2901 \text { from MH \& } 403 \text { from MHC. Data from patient information }\end{array}$ \\
\hline $\begin{array}{l}\text { Key words: } \\
\text { Adverse drug reactions, } \\
\text { Antiretroviral drugs. }\end{array}$ & $\begin{array}{l}\text { sheet were collected using pre-tested data collection format from April to May, 2009. Of the study subjects } 1597 \& \\
234 \text { were females from MH \& MHC respectively. The regimens used during the study period were ZDV/3TC/NVP, } \\
\text { D4T/3TC/NVP, D4T/3TC/EFV and ZDV/3TC/EFV. Occurrence of ADR was the reason for change in regimen in } 255 \\
\text { of the total } 3304 \text { patients; the prevalence of which was } 8.31 \% \text { in MH and } 3.47 \% \text { in MHC. The most common adverse } \\
\text { reactions recorded were peripheral neuropathy, anemia \& lipodystrophy in MH and anemia, skin rash \& liver toxicity } \\
\text { in MHC. High correlation was observed between being female and the occurrence of ADRs. This study has shown that } \\
\text { the occurrence of ADR is a significant factor in changing regimens in health care facilities in Mekelle. }\end{array}$ \\
\hline
\end{tabular}

\section{INTRODUCTION}

Few times before having AIDS was considered as equivalent to death. But after the introduction of combined anti retro viral therapy (ART) since 1996, AIDS has become chronic manageable disease (Hammer et al., 2006). Combination ART prolongs life and prevents progression of the disease caused by HIV. Currently there are more than 20 FDA approved ARVs in the world with different classes that act differently at different stages of HIV life cycle (Charles, 2005).

Although highly active antiretroviral therapy (HAART) has changed the natural history of HIV infection, the presences

\footnotetext{
"Corresponding Author Lecturer of Pharmacology, Department of Pharmacology and Clinical Pharmacy, School of Pharmacy, College of Health Sciences, Addis Ababa University Addis Ababa, P.O. Box 1176, Ethiopia. E-mail:abebe.ejigu@aau.edu.et; hailupharma@gmail.com
}

of adverse events have limited its efficacy. Almost all ARV drugs have some adverse effects which can be specific to a single drug or shared by class of drug or could be shared by all ARV drugs. According to the World Health Organization definition ADR is any noxious, unintended, and undesired effect of a drug, which occurs at doses used in humans for prophylaxis, diagnosis, or therapy. This definition excludes therapeutic failures, intentional and accidental poisoning (i.e., overdose), and drug abuse (Fauci and Lane, 2005; Kumarasamy et al., 2006).

Drug toxicity is the main reason for treatment change (Bratt et al., 2001) and also reported as one of the main difficulties for patients using ART. Moreover, ADRs are one of the leading causes of morbidity \& mortality in the health care. The institute of medicine reported in Jan 2000 that from 44000 to 98000 deaths occur annually from medical errors of this total, an estimated 7000 death due to ADRs. Studies on the incidence of serious ADRs estimate that $6.7 \%$ of hospitalized patients have a serious ADR 
with fatality rate of $0.32 \%$ \& also estimated that over 350000 ADRs occur in US nursing homes (John et al., 2001). For the case of ARV ADR about $25 \%$ of the patients stop therapy with in the first year on HAART because of side effects and about the same number of patients does not take the recommended dosages of their medication due to the concern of side effects. The majority of adverse effects \& toxicities that are known to HIV treatment are those derived from cohort studies or clinical trials of patients in the wealthier nations like North America, Europe and Australia, and based on innovator drug products. But because of influences of environment, co infection, host genetics, poor nutritional status $\&$ other factors the adverse effect profile in Africa may differ (Carr and Cooper, 2000). The main aim of the present study was to determine the prevalence of HAART ADRs on HIV positive patients in Mekelle health facilities.

\section{METHODOLOGY}

The study was conducted at Mekelle Hospital (MH) \& Mekelle Health center (MHC) of Mekelle town, which is located 783 KM North of the capital, Addis Ababa in Tigray regional state. $\mathrm{MH}$ is a 200 bed general hospital serving for a population of Mekelle \& other neighboring cities. The hospital has different departments and provides general out patient service, surgical \& obstetric emergency \& regular services, general medical \& pediatric inpatient services, HIV counseling \& testing. It is staffed with $212 \& 102$ technical \& administrative staffs respectively. The hospital has been providing HIV counseling \& testing service since July 2003 with a charge made by the hospital to very few HARRT users. Similarly MHC is also a health institution offering service for people around Mekelle. It has no beds for inpatients as a result serious cases were referred to other higher level health institutions. As in the case of $\mathrm{MH}$, the HIV counselling and testing service is given in this facility since January 2007 and it is providing HAART for more than $400 \mathrm{HIV}$ patients.

The study was conducted from April to May, 2009. A cross-sectional study design based on HAART patient's record review was employed to determine the prevalence of ADR and those entire patient information sheets (PIS) card in ART pharmacy were used to collect the data. All patients on HAART who were active and took HAART for at least a month were included in the study. Patients who had lost follow-up, transferred to other health facilities, died and who had taken the medication for the first time only were excluded from the study. Information sheet was used to collect data on age, sex, weight, time of exposure, regimen for ARV, concomitant disease, drugs for opportunistic infection, from PIS card in ART Pharmacy, eligible date and date during ADR and regimen of during ADR, history of ADR retrospectively. All the necessary information obtained from the patient information sheet were recorded \& documented. Data was checked for completeness and errors in data collection, recording, entry and coding. It was explored for checking outliers, extreme values, and normality of the distribution. The data was analyzed using SPSS version 20.

An ethical approval was obtained from the department of Pharmacy, Mekelle University and an official letter was sent from the department to each health facilities. Oral consent was obtained from data clerks and ART pharmacist. Patient identifiers were excluded from the data collection format or any information which can identify a particular patient like name and card number was excluded from the data collection format.

\section{RESULTS}

\section{Characteristic of study population}

Data were collected from a total of 2901 and 403 HAART patients' information sheet records in $\mathrm{MH}$ and $\mathrm{MHC}$ respectively during the study period as described in Table 1. Among the total study subjects in MH 1304 (44.95\%) were male and $1597(55.05 \%)$ female with weight ranging from 4.2 to $85 \mathrm{Kg}$. Moreover, 123 (4.24\%) of HAART patients in MH were children below five years while $132(4.55 \%)$ were children between five and twelve years. Similarly, majority of the study subjects in MHC were female accounting for 58.06\% (234) while 169 (41.94\%) were males with weight range of 31 to $75.5 \mathrm{Kg}$. However, there were no eligible children under 12 years in MHC.

Table 1: Distribution of HAART patients by age and sex in MH and MHC from April to May, 2009.

\begin{tabular}{|c|c|c|c|c|c|}
\hline \multirow{2}{*}{ Health Facility } & \multirow{2}{*}{ Sex } & \multicolumn{4}{|c|}{ Age of patients (year) } \\
\hline & & $<5$ & $5-12$ & $>12$ & Total (\%) \\
\hline \multirow{3}{*}{$\mathrm{MH}$} & Male & 61 & 60 & 1183 & $1304(44.95 \%)$ \\
\hline & Female & 62 & 72 & 1463 & $1597(55.05 \%)$ \\
\hline & Total & $123(4.24 \%)$ & $132(4.55 \%)$ & $2646(91.21 \%)$ & $2901(100 \%)$ \\
\hline \multirow{3}{*}{ MHC } & Male & - & - & 169 & $169(41.94 \%)$ \\
\hline & Female & - & - & 234 & $234(58.06 \%)$ \\
\hline & Total & - & - & $403(100 \%)$ & $403(100 \%)$ \\
\hline
\end{tabular}

\section{Antiretroviral regimen}

The antiretroviral regimen of the patients during the data collection period is depicted in Table 2. Most of the patients in $\mathrm{MH}$ and all patients in $\mathrm{MHC}$ were under fist line regimen. ZDV/3TC/NVP was the most prescribed regimen in $\mathrm{MH}$ accounting for $37.40 \%$ followed by $\mathrm{D} 4 \mathrm{~T} / 3 \mathrm{TC} / \mathrm{NVP}$
(37.09\%), D4T/3TC/EFV (11.27\%) and ZDV/3TC EFV $(8.82 \%)$. While the orders in the MHC were D4T/3TC/NVP (43.42\%), ZDV/3TC/NVP (21.59\%), D4T/3TC EFV (29.03\%) and $\mathrm{ZDV} / 3 \mathrm{TC} / \mathrm{NVP}(21.59 \%)$. However, few patients in $\mathrm{MH}$ were under second line regimen which was totally absent in MHC. 
Table 2: Current regimen of patients on ART in MH and MHC, April to May, 2009.

\begin{tabular}{ccccc}
\hline \multirow{2}{*}{ ART regimen } & \multicolumn{2}{c}{ MH } & \multicolumn{2}{c}{ MHC } \\
\cline { 2 - 5 } & Total & Percent (\%) & Total & Percent (\%) \\
\hline ZDV/3TC/NVP & 1085 & 37.40 & 87 & 21.59 \\
ZDV/3TC EFV & 256 & 8.82 & 24 & 5.96 \\
D4T/3TC/NVP & 1076 & 37.09 & 175 & 43.42 \\
D4T/3TC EFV & 327 & 11.27 & 117 & 29.03 \\
TDF/3TC NVP & 91 & 3.13 & - & - \\
TDF/3TC EFV & 31 & 1.07 & - & - \\
TDF/ABC/LOP/r & 21 & 0.72 & - & - \\
ZDV/3TC/LOP/r & 3 & 0.10 & - & - \\
TDF/3TC/LOP/r & 6 & 0.21 & - & - \\
D4T/3TC/LOP/r & 2 & 0.07 & - & - \\
TDF/ABC/NVP & 1 & 0.03 & - & - \\
TDF/DDI/LOP/r & 1 & 0.03 & - & - \\
ABC/DDI/LOP/r & 1 & 0.03 & - & - \\
TOTAL & 2901 & 100 & 403 & 100 \\
\hline
\end{tabular}

\section{Occurrence of ADRs}

The occurrence of ADRs in MH is shown in Figure 1. Among the total 2901 patients on HAART in MH, $241(8.31 \%)$ ARV treatment related ADRs which make the patient to change regimen were found. The predominant ARV related ADRs in $\mathrm{MH}$ were peripheral neuropathy accounting for $45.23 \%(\mathrm{n}=$ $109)$ followed by anemia $(29.04 \%)$, lipodystrophy $(8.71 \%)$, skin rash $(7.88 \%)$ \& liver toxicity $(7.05 \%)$. Other ADRs which result in treatment alteration but with less frequency were severe pain in $1.66 \%$ and CNS side effect in $0.41 \%$ of the patients in $\mathrm{MH}$. In almost all cases of the ADR females were more affected than men's in MH. Peripheral neuropathy (57.80\%), anemia (58.57\%), skin rash $(57.89 \%)$, liver toxicity $(58.82 \%)$ and lipodystrophy (76.19\%) ADR were the ADRs experienced by females.

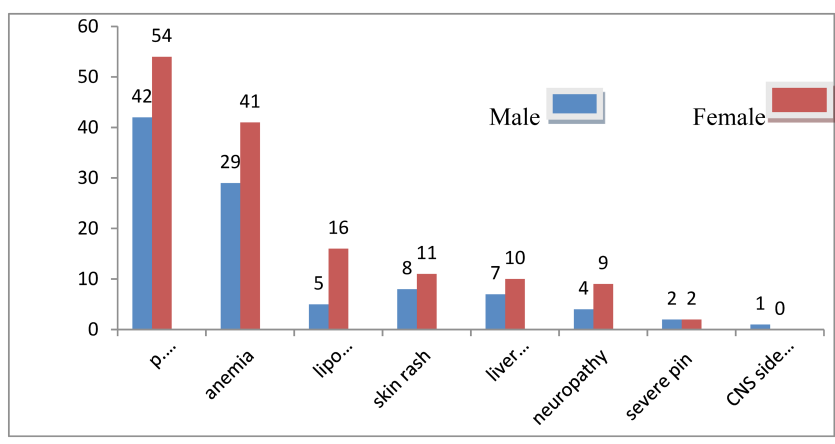

Fig. 1: The distributions of HAART ADRs by sex in MH from April to May, 2009.

Unlike MH very few cases and number of ADR were observed in MHC. Only $3.47 \%$ of the patients experienced ADR resulting in treatment alteration. The ADRs were anemia in $42.86 \%$, skin rash in $35.71 \%$, liver toxicity in $4.28 \%$ and peripheral neuropathy in $0.71 \%$ of the patients (Figure 2). But the prevalence of ADR based on sex in was almost the same except for skin rash in which females were more affected than males (4 cases from the total of 5 cases of skin rash).

\section{$A D R$ in relation to Age}

Table 3 shows the distribution of ADRs in relation to age in $\mathrm{MH}$ only as pediatric dosage forms were absent in MHC. As depicted in the table among the total of 241 (8.31\%) cases of ADR in $\mathrm{MH}$, only 16 cases $(0.66 \%)$ were attributed to children of age below 12 years. Of this 8 occurred in age $5 \&$ below while the other eight cases occurred between age 5 and 12 years. The predominant ADR observed in children was anemia accounting for 12 cases $(80 \%)$ from the total 16 cases. Other Adverse effects observed include lipodystrophy, liver toxicity, neuropathy, and CNS side effects.

Table 3: the occurrence of ADR by age in MH April-May, 2009.

\begin{tabular}{|c|c|c|c|c|c|c|}
\hline \multirow{2}{*}{ Type of ADR } & \multicolumn{2}{|c|}{$<5$} & \multicolumn{2}{|c|}{$5-12$} & \multicolumn{2}{|c|}{$>/ 2$} \\
\hline & Number & Percent $\%$ & Number & Percent $\%$ & Number & Percent $\%$ \\
\hline Peripheral Neuropathy & 1 & 0.92 & - & & 108 & 99.08 \\
\hline Anemia & 6 & 8.57 & 6 & & 58 & 82.86 \\
\hline Lipodystrophy & - & - & 1 & & 20 & 95.24 \\
\hline Skin rash & - & - & - & & 19 & $100 \%$ \\
\hline Liver toxicity & - & - & 1 & & 16 & 94.12 \\
\hline Sever pain & - & - & - & & 4 & $100 \%$ \\
\hline CNS side effect & 1 & $100 \%$ & - & & - & - \\
\hline Total (\%) & 8 & 3.32 & 8 & & 225 & 93.36 \\
\hline
\end{tabular}

\section{Regimen during ADR}

The regimens during the occurrence of ADR are shown in Table 4 below. The predominant regimens during ADR were D4T/3TC NVP and D4T/3TC EFV in case of peripheral neuropathy accounting for $89(81.65 \%)$ and 20 $(18.35 \%)$, in lip dystrophy $16(17.19 \%) \& 5(23.81 \%)$ of the cases respectively. ZDV/3TC/NVP and ZDV/3TC/EFV were dominant in case of anemia $(58(82.86 \%) \& 6(8.57 \%))$ and liver toxicity $(11(64.70 \%)$ and $1(5.88 \%))$. ZDV/3TC/NVP and D4T/3TC/NVP which are NVP based regimens were responsible for the occurrence of skin rash. Similar results were obtained in MHC. 


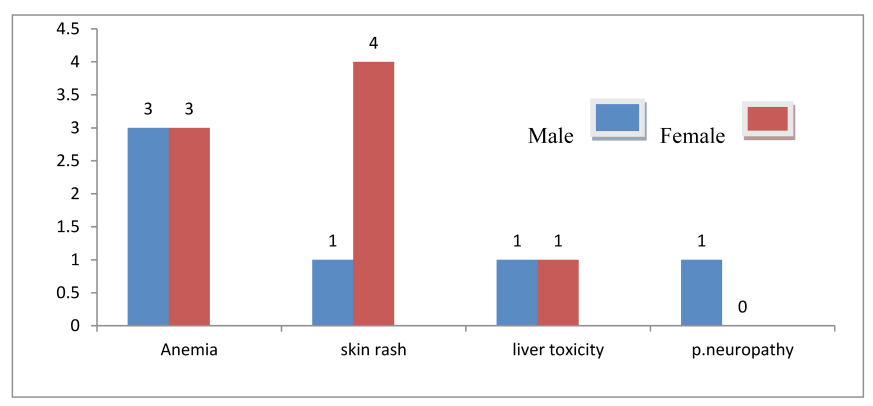

Fig. 2: Distribution of HAART ADR in relation to sex in MHC, April to May, 2009.

The time gap between eligibility and experience of ADR was different depending on the type of ADR. Peripheral neuropathy in most patients $(69.79 \%)$ occurred after 1 year while skin rash occurred almost in all cases (18 cases among 19) before 2 months of which all except 2 were occurred within a month from this also except the two which occur within 2 month, all others occur within in a month. The other ADRS like liver toxicity and anemia occurred mostly before 5 months, for lipodystrophy after 9 month (19 of the cases) and after 1 year for severe pain and CNS side effect. In the same manner all cases of anemia occurred before eight months, skin rash before four months, and liver toxicity before two months and peripheral neuropathy within 9 months in MHC.

Moreover, it was from the data collection format that TB was recorded as concomitant disease among 15 patients on HAART in MH, 8 in peripheral neuropathy experienced patients, 6 in anemia and 1 in liver toxicity but only one TB case in MHC in patient who experienced skin rash. Almost, all patients in both facilities used Cotrimoxazole for prophylaxes. Other OI drugs other than those anti-TB and Cotrimoxazole were not recorded well.

Table 4: The relation between occurrence of ADR and HAART regimen in MH and MHC, April to May, 2009.

\begin{tabular}{|c|c|c|c|c|c|c|}
\hline \multirow{2}{*}{ Type of ADR } & \multirow{2}{*}{$\begin{array}{l}\text { Heath } \\
\text { facility }\end{array}$} & \multicolumn{5}{|c|}{ Regimen during ADR } \\
\hline & & $1 \mathbf{a}$ & 1b & $1 \mathrm{c}$ & 1d & 1e \\
\hline \multirow{3}{*}{ Anemia } & $\mathrm{MH}$ & 3 & 3 & 55 & 9 & - \\
\hline & MHC & - & - & 3 & 3 & - \\
\hline & MH & 3 & - & 16 & - & - \\
\hline Shin rash & MHC & 3 & - & 2 & - & - \\
\hline \multirow{2}{*}{ Liver toxicity } & MH & 5 & - & 11 & 1 & - \\
\hline & MHC & 2 & - & - & - & - \\
\hline \multirow{2}{*}{ Peripheral neuropathy } & MH & 89 & 20 & - & - & - \\
\hline & MHC & 1 & - & - & - & - \\
\hline Lipodystrophy & MH & 16 & 5 & - & - & - \\
\hline Severe pain & MH & - & - & 3 & - & 1 \\
\hline CNS side effect & MHC & - & - & - & 1 & - \\
\hline Total & & 122 & 28 & 90 & 14 & 1 \\
\hline
\end{tabular}

$\mathrm{NB} ; 1 \mathrm{a}=\mathrm{D} 4 \mathrm{~T} / 3 \mathrm{TC} / \mathrm{NVP} ; 1 \mathrm{~b}=\mathrm{D} 4 \mathrm{~T} / 3 \mathrm{TC} / / \mathrm{EFZ} ; 1 \mathrm{c}=\mathrm{ZDV} / 3 \mathrm{TC} / \mathrm{NVP} ; 1 \mathrm{~d}=$ ZDV/3TC/EFZ; 1e = TDF/3TC/NVP.

\section{DISCUSSION}

ADRS to HAART are the main causes of non-adherence and treatment changes as described by many studies (Alemayehu et al., 2008; Alexander et al., 2001). The prevalence and frequency of ADR could vary based on pharmacogenomics, sex, stage of HIV and in general from country to country (Makradi and Mafatia, 2005).

In this study the overall prevalence of ADR was found to be $7.72 \%$ in which $8.31 \%$ in $\mathrm{MH}$ and $3.47 \%$ in $\mathrm{MHC}$, which is by far very less than other studies done in other countries and in other health care facilities in Ethiopia. A Swaziland cohort study found the prevalence to be $20.3 \%$ (Alexander et al., 2001), an Indian study conducted on 300 patients receiving NVP or EFV based regimens found the prevalence to be $15.5 \%$ patients to change at least one drug from the previous regimen on account of toxicity (Wanchu et al., 2007). An Ethiopian study at Zewditu Memorial hospital founds the prevalence of ADR to be $18.44 \%$, which is responsible for treatment change (Assegid, 2007). In the current study ADR was taken from recorded PIS and ADR is recorded in the study areas only when there is treatment change to ARVs. But medical record adverse events are different from those reported by the patients. A study by Epstein et al. (2008), in Massachusetts Hospitals has shown that from 998 study patients, $23 \%$ had at least one adverse event detected by interview and $11 \%$ had at least one adverse event identified by record. This indicates that there were 21 serious and preventable adverse events that were not documented in a medical record. By this study patient reported ADR was not obtained because no questioner was prepared. This might be one reason for the low prevalence of ADR in the study areas than other studies. Although studies done in clinical records have found high prevalence of ADR, like in Zewditu Memorial Hospital (Assegid, 2007), but this study differs from others in that no sampling was made to include in the study population. This will have an effect on the prevalence.

In addition, there is a need for intense laboratory monitoring to diagnose drug toxicity early besides the clinical diagnosis. But this might depend on the health care infrastructure and patients' awareness to report ADRs early. In resource poor countries like the study areas ADRs are mostly obtained from clinical diagnosis and patients have poor awareness regarding medication side effects. (Tesiorowski et al., 2002).

Moreover, ADRs are one of the reasons for loss of follow up of HAART and deaths as well. In this study PIS of those who have lost follow up and who have died was not reviewed. This and the relative dynamic nature of ADR and other reasons might result in the low prevalence of ADR in the study areas. But, it should be reminded that the continual monitoring of ADR and its determinants in Ethiopia should not be undermined and require further study.

The most commonly recorded ADRs in the study areas were peripheral neuropathy, anemia, and skin rash in general and peripheral neuropathy, anemia and lipodystrophy in $\mathrm{MH}$, whereas anemia, skin rash and liver toxicity in MHC. The drugs mostly associated for the potential ADRs were D4T for peripheral neuropathy and lipodystrophy, ZDV for anemia and NVP for skin rash and liver toxicity. These results are consistent with most studies. A study in Arbaminich hospital among 124 
episodes of drug related side effects; the most prevalent were peripheral neuropathy $(38.7 \%)$, skin rash $(33 \%)$ and anemia $(8.9 \%)$ (Degu, 2006). From a study among 300 patients in India, lipodystrophy $(31.91 \%)$, skin rash $(21.28 \%)$ and peripheral neuropathy $(12.76 \%)$ were common among others (Wanchu et al., 2007). From a study among three resources limited setting neuropathy, neutropenia and lipodystrophy were dominant in Africa (WHO, 2008), which is consistent with the result in the hospital. But it is difficult to put clear cut comparisons to the prevalence of specific ADR of HAART in different studies because the occurrence of ADR will depend on different factors. A common methodological frame work will be needed to assess and compare the extent and nature of toxicities in various setting. The different levels of health care system have varying infrastructure to assess and conduct diagnostic procedure needed for adverse events.

For example, when we compare the two health facilities i.e. $\mathrm{MH}$ and $\mathrm{MHC}$ in this regard. The number and over all prevalence of ADRs is very low in MHC than that of MH. This might be attributed to the difference in qualified professionals and different diagnostic methods to detect ADR among other reasons. The health center described here provides a large service by nurses and pharmacy technicians without appropriate physician and pharmacist supervision. Only one nurse and one pharmacy technician are involved in prescribing and dispensing of ARV with appropriate counseling respectively. Capitalizing the potential of nurses and pharmacy technicians would be necessary because it is difficult to achieve ART care only relying on physicians and pharmacists. It is better that the complementary between all health professionals' skills has been important to achieve ART service well. The unavailability of second line drugs might also be the other factor that led to low prevalence of ADR in health center. All the ADR in this study are those that result in treatment alterations, but if second line drugs are not present patients might be compelled to continue on their present regimens.

One factor that is used for deciding ADR is sex. Many studies revealed that female HIV patients have higher propensity of developing skin rash and syptomatic hepatic events from NVP (Makrandi and Marfatia, 2005). An Indian study have shown females to be more affected than males in developing rash, $14.7 \%$ and $10.5 \%$ respectively. A cohort study in Dutch hospitals also put female sex (39\% higher risk of developing hyper sensitivity reaction than males) to be an independent risk factor for ADR (Anouk et al., 2008). Among 11 participants experiencing drug related toxicity, $90 \%$ occur in women (Mayer et al., 2009). In this study also there was high relation between being a female and occurrence of ADR. 59.75\% $(n=144)$ of the cases among the total $241 \mathrm{ADR}$ cases in $\mathrm{MH}$ and 57.145 $(\mathrm{n}=8)$ of the ADR cases in MHC were attributed to females. Among the ADRs, lipodystrophy (76.19\%) in $\mathrm{MH}$ and skin rash $(80 \%)$ in $\mathrm{MHC}$ were dominant in females. Consistent findings were also reported in comparable studies (Bianchi et al., 2009). Final differences in drug levels or in drug response can depend on sociocultural, psychoperceptual and behavioural factors, body size and composition, genetic, molecular or biochemical factors and hormonal/reproductive influences (Ofotokun and Pomeroy, 2003). But it is difficult to generalize based on this study as females are more affected than males in all ADRs. This is because to have such kind of generalization the number of females and males in the study should be equal, but in these study females cover most of the study population in both $\mathrm{MH}$ and $\mathrm{MHC}$.

Few studies have evaluated the relation between time of exposure and occurrence of ADR (Assegid, 2007; Degu, 2006). Anemia was the easiest side effect occurring at a median time of 4.4 weeks followed by skin rash occurring at median of 4.6 weeks in Arbaminch hospital (Degu, 2006). In a study at Zewditu Memorial hospital, peripheral neuropathy was diagnosed beyond 6 month duration and commonly after 15 months, while anemia was reported throughout treatment duration (Assegid, 2007). Other studies show occurrence of NVP induced rash to occur in the first six weeks (Ajay, 2007; Chatiya, 2007). Serious toxicities due to HAART in this study occurred as early as two weeks in the case of skin rash and as large as 48 weeks as in the case of peripheral neuropathy. The result to know the relation between ADR and time of exposure will depend on the time to diagnose drug toxicities early using laboratory monitoring (anemia while the patient on ZDV and hepatitis while on NVP). Laboratory monitoring as been told in the hospital is done in the first three months of therapy and not continuous after that, meaning there is high time gap given to do laboratory for each patients after three months. So cases that are not detected in the first three months will wait the next laboratory monitoring even if they occur before that time. And also patients may not be aware of that symptoms that occur during HAART are medication side effects by accepting that ADR due to physical, social and emotional morbidity associated with AIDS patients. So some patients report early while others late after occurrences of ADR when repeated. In this regard, it is difficult to find substantial evidence to suggest that prolonged exposure is associated with ADR since this will depend on the patient and the type of ADR to be reported. But in general it can be said based on the result as other studies skin rash and liver toxicity occur early after initiating HAART especially skin rash after one month of treatment. While peripheral neuropathy and lipodystrophy occur after long time and thus they can be associated with prolonged exposure or have late onset as compared to others.

The other finding in this study is the occurrence of anemia, liver toxicity and other adverse effects in children's of age 12 and below, which is consistent with study in South America, which noted anemia, gynacomastia and hepatitis to be common in children's of age less than 13 years (WHO, 2008). The prevalence of HAART ADR in children was found to be only 16 cases out of the total 255 children's of age 12 and below (6.67\%) in $\mathrm{MH}$. Even though few studies have been done on the prevalence of ADR in children's, the result obtained in this study is lower than a study in Rwanda which is 8.3\% (Anad et al., 2007), and that of South Africa which was found to be $11.3 \%$ (Asimwe et al., 2007). Diagnosing ART toxicity in children might be more difficult because children can't express themselves well as adults. Those ADRs which need laboratory monitoring like anemia and liver toxicity are reported in most cases. Thus additional monitoring and qualified professionals might be needed to improve the detection rate of ADR in children. This highlights the need for closer attention to be paid to children in monitoring the ADRs associated with HAART. 
Some of the ADRs could be associated with other drugs used by the patients for the treatment of opportunistic infections or can be the symptoms of the diseases or other concomitant diseases occurring in these patients. One study in pre-HAART HIV positive patients shows hepatotoxicity to be one of the important ADR associated with anti TB drugs (Getnet, 2008). Isoniazid is also associated with peripheral neuropathy. In this study eight patients who have experienced peripheral neuropathy have taken anti TB drugs but no person with liver toxicity have taken anti TB as obtained from the record. But it is difficult to relate these things because the times where the anti TB drugs are taken were not recorded, so it might be before the occurrence of ADR or after that. And also this anti-TB drugs were recorded only when the patients changes regimen from NVP to EFV. In general there is poor recording of other drugs other than HAART and other concomitant diseases in both facilities. But cotrimoxazole is prescribed to almost all patients but this drug is associated with gastrointestinal side effects which are not observed in our result. The reason for this poor recording is, only few opportunistic drugs are available in ART pharmacy and patients are obligated to buy from retail pharmacy of the hospital or in other private pharmacies. The other reason is the imbalance of the professionals to that of the tasks performed in the ART pharmacy. Only one pharmacy technician in each facility are performing the bulk activities in ART. Thus it would be said that improving the ADR recording profile and increasing the number of professionals will be important to have good monitoring of ADR.

Despite these findings there are some limitations to the study. The main limitation of the study is patient reported ADR were not included and the study does not include those who have lost follow up and died, so difficult to know the actual prevalence of ADR in the facilities. The study was conducted in only two health facilities therefore some of the findings might not be generalized to setting such as referral hospitals and other health institutions with fewer or higher programs. But the main strength of the study is being conducted without sampling.

\section{CONCLUSION}

This study has shown that the occurrence of ADR is a significant factor in changing regimens in health care facilities in Mekelle. It, hence, highlights the necessity for health care providers to be equipped with knowledge of ADRs with HAART regimens; patients at risk, frequency of occurrence, and their management in preference to and/or in addition to changing the regimen.

\section{ACKNOWLEDGMENT}

Financial support and sponsorship: We would like to acknowledge the financial support of the Mekelle University.

\section{CONFLICT OF INTERESTS}

There are no conflicts of interest.

\section{REFERENCES}

Ajay S, Archana S, Megha M, Marfatia Y. Cutaneous eruptions associated with NVP therapy. Indian J Sex Transm Dis, 2007; 28(2):94-
96

Alemayehu A, Belaineh G, Kebede D, Kifle W, Sofonias G. Predictors of Adherence to antiretroviral therapy among HIV infected persons: a prospective study in Southwest Ethiopia. BMC Public Health, 2008; 8: 265.

Alexander H, Heiner CB, Reto N, Manuel B. Reasons for Discontinuetion of first HAART in a cohort of protease inhibitor naïve HIV infected. J AIDS, 2001; 26(2).191-193.

Anad R, Dorse GL, France H, Geddes R, Giddy J, Grobler AC, Helga H, Leeper SC, Mntambo M, Nixon K, Thomas M, Vlok W. Preliminary outcomes of a pediatric highly active Antiretroviral Therapy cohort from Kwazulu-Natal, South Africa. BMC Pediatr, 2007; 7:13.

Anouk M, Brinkman K, Feridinand Gras L, Joep M, Marchina F, Reiss P, Richte C. Discontinuotion of NVP because of hyper sensitivity reaction in patients with prior treatment experienced compared with treatment naïve patients Athena Cohort study. CID, 200; 846 (15): 933-940.

Asimwe A, Gazille C, Griensven J, Naeyer L, Reid T, Uwera J. Success with antiretroviral treatment for Childrens in Kigali, Rewanda: Experience with health center/nurse-based care. BMC pediatr, 2007; 8:39.

Assegid T (2007). Assessment of the prevalence of adverse drug reaction among HIV infected adults on antiretroviral therapy and factors associated with it at Zewditu memorial Hospita. Addis Ababa Univerity (thesis).

Bianchi L, Blanch J, Blanco J, Conget I, Cuevas PJ, Garcia M, Mallolas J, Martinez E, Mocroft A, Philips A. Risk of Lipodystrophy in HIV 1 infected patients treated with protease inhibitors: a prospective cohort study. Lancet, 2009; 357 (9256): 592-598.

Bratt G, Koppel K, Sandstorm E. The development of laboratory cardiovascular risk factors in patients on HAART during 24 months followup. Inter Medic press, 2001; 6(14): 52.

Carr A, Cooper DA. Adverse effects of antiretroviral therapy. Lancet, 2000; 356:1423-30.

Charles F (2005). Antiretroviral agents and treatment of HIV infection in: Goodman and Gilman's the Pharmacological basis of therapeutics. Brunton L., Lazo J., and Parker KL. (eds), $11^{\text {th }}$ ed, Mc Graw Hill, Newyork 1273-1314.

Chatiya A, Weerawat M. Adverse drug events and efficacy of ART among HIV infected patients concurrently receiving NVP based ART and Fluconazole. BMC Infect Dis, 2007; 7:14.

Degu J, Lindtjorn B, Naess A. Antiretroviral therapy at a district hospital in Ethiopia prevents death and tuberculosis in a cohort of HIV patients UK. AIDS Res Thera, 2006; 3:10.

Epstein AM, Schneider EC, Weingart S, Weisman J. Comparing patient reported Hospital Adverse with medical review. Imp Pt Care, 2008; 149 (2):100-108.

Fauci AS, Lane CH. (2005) Human Immunodeficiency virus disease AIDS and related disorders. In Harison's principle of medicine Kasper Dennis L., Longo DL., Braunwald E., Hauser SL., Jomson LD. (eds), $16^{\text {th }}$ ed. Mc Grraw Hill, Newyork 1071-1139.

Getnet Y, Getachew A, Wondwossen A, Eyasu M, Eleni A, Lars L, Lawrence Y, Beniyam F, Abraham A. Anti tuberculosis therapy induced hepatotoxicity among Ethiopian HIV positive and negative patients. PLOs One, 2008; 3(3):1809.

Hammer SM, Saag MS, Schechter M, Montaner JS, Schooley RT, Jacobsen DM, et al. Treatment for adult HIV infection: recommendations of the International AIDS Society-USA panel. JAMA, 2006; 296:827-43.

John M, Moore CB, James IR, Nolan D, Upton RP, McKinnon EJ, et al. Chronic hyperlactatemia in HIV-infected patients taking antiretroviral therapy. AIDS, 2001; 15:717-23.

Kumarasamy N, Vallabhaneni S, Cecelia AJ, Yepthomi T, Balakrishnan P, Saghayam S, et al. Reasons for modification of generic highly active antiretroviral therapeutic regimens among patients in southern India. J Acquir Immune Defic Syndr, 2006; 41:53-8.

Makrandi S, Marfatia S. Adverse drug reactions due to ARV, Issues and Challenges. J Sex Transm Dis, 2005; 26(2): 40-49. 
Mayer L, Pitt J, Wood R. Quality of life and the impact of drug toxicities in a South African community based antiretroviral programme. J. Inter AIDS Society, 2009; 12(5).

Ofotokun I, Pomeroy C. Sex Differences in Adverse Reactions to Antiretroviral drugs. Inter AIDS Society, 2003; 11(2).

Tesiorowski AM, Harris M, Chan KJ, Thompson CR, Montaner JSG. Anaerobic threshold and random venous lactate levels among HIV-positive patients on antiretroviral therapy. J Acquir Immune Defic Syndr, 2002; 31(2):250-1.

Wanchu A, Kaur R, Bambery P, Singh S. Adherence to generic reverse transcriptase inhibitor-based antiretroviral medication at a Tertiary Center in North India. AIDS and Behavior, 2007; 11(1):99-102.
WHO. 2008. ARV drugs Adverse events, case definition, grading laboratory diagnosis and treatment monitoring. http://www.hivforum. org (Accessed at January 10, 2009).

How to cite this article:

Ejigu A, Gehzu M, Haileselassi W. Adverse drug reactions causing treatment change among patients taking highly active antiretroviral therapy in health care facilities of Mekelle, Ethiopia. J App Pharm Sci, 2018; 8(03): 104-110. 